\title{
PANEL DISCUSSION ON THE MANAGEMENT OF ALLERGIES IN GERIATRIC PATIENTS*
}

Moderator: John M. Sheldon, M.D. Professor of Internal Medicine, and Director, Department of Post-Graduate Medicine, University of Michigan Medical School, Ann Arbor, Michigan.

Panelists: Romert A. Cooke, M.D., Director, Institute of Allergy, Roosevelt Hospital, New York, N. Y.

Stanley G. Hampton, M.D., Assistant Clinical Professor of Medicine, and Director, Allergy Clinic, Washington University School of Medicine, St. Louis, Missouri.

Bram Rose, M.D., Associate Professor of Medicine, McGill University, and Associate Physician, Royal Victoria Hospital, Montreal, Canada.

William B. Sherman, M.D., Associate Clinical Professor of Medicine, Columbia University, College of Physicians and Surgeons, New York, N. Y.

Moderator John M. Sheldon: I shall ask the panelists a number of questions relative to the problem of allergy and pertinent to the senior citizens of our country. Because of their importance, we shall attempt to emphasize asthma and conditions of wheezing and dyspnea. We hope, time permitting, to remark upon other allergic manifestations in geriatric patients.

First, I want to ask Dr. Cooke about the effect of aging upon pre-existing allergic disease, particularly asthma, hay fever, and dermatitis. What is your view, Dr. Cooke?

Dr. Robert A. Cooke: When we speak of allergic diseases, we should preface our remarks by saying that, even today, it is almost impossible to express a positive opinion on any phase of allergic phenomena that cannot be disputed. However, I shall try to give what I believe to be the consensus with regard to certain points.

Basically, there is evidence that allergies develop as a result of some hereditarily transmitted capacity, which we speak of in general terms as an "allergic constitution." When we say that, we mean only that persons who acquire allergies have a capacity to react in a way that normal and nonallergic persons do not.

To put it another way, substances with which one comes in contact in any way externally, or by inhalation, ingestion, injection or infection, may react to produce allergic lesions such as asthma, hay fever, dermatitis or urticaria.

The Moderator has asked: "What is the effect of aging upon such diseases?" In infancy, the principal allergies, aside from an occasional acute one due to food, are dermatitis (eczema) and asthma. As eczematous infants grow older, at about 5 years of age or earlier, the eruptions tend to diminish and undergo spontaneous cure. However, because these children have an inherited allergic constitution, asthma and hay fever often develop, with sensitivity to dusts, pollens or animal danders by inhalation or possibly by infection. For the most

* Conducted at the Graduate Symposium on Geriatric Medicine of the American Geriatrics Soeiety and the American Academy of General Practice (Rhode Island and New York Chapters), New York City, November 19 and 20, 1956. 
part, they carry this allergy through life, but as they continue aging something new may happen. When they reach the age of 40 or so, infection becomes a major factor in accentuating the symptoms; it may even be a cause, as bacteria can act as allergens. This is known as bacterial allergy.

In cases of asthma which begin in childhood and continue up to about age 40 , the basic sensitivity to inhaled substances is combined with the factor of infection. However, asthma which begins after age 40 is likely to represent bacterial allergy, and requires prompt and effective treatment; otherwise, it will continue with gradually increasing severity throughout life. The same is true of vasomotor rhinitis. Although I have seen hay fever from pollen or dust begin as late as age 70 , onset at such a late age is unusual. There is something special in the way of an inherited constitutional defect in all allergies. Once started, allergy continues through life, manifesting itself in one form or another. The earlier the cause is discovered and overcome, the less disability there will be. The allergic person may live with his sensitizations, but devoid of symptoms, as long as he has no contact with the sensitizing substances-dusts, foods, drugs or infections.

Moderator Sheldon: Thank you, Dr. Cooke. Has any other member of the panel a comment with regard to the effect of aging upon pre-existing allergic disease?

Dr. Hampton, I should like to ask you a specific question. What is the effect of aging upon the allergenic reaction to a test on the skin?

Dr. Stanley G. Hampton: The reaction to a skin test in the young and the middle-aged adult is rather outstanding. The skin of an aged person does not react to the test as intensively as that of a younger person. There are 2 parts to a positive skin reaction-the wheal and surrounding erythema. In elderly people, the wheal may occur as in younger persons, but the erythema may be much less intense. This tends to support the idea of a triple response as proposed by Lewis: 1) increased permeability of capillaries accounts for the wheal, 2) dilatation of the capillaries accounts for the immediate surrounding erythema, and 3) dilatation of the afferent arterioles accounts for the red flare. Perhaps, because of the less elastic capillaries and arterioles of the aged skin, the second and third phases of the triple response are reduced, thus rendering the skin less reactive.

One piece of investigative work does not seem to confirm this clinical experience. The work was carried out a few years ago at the Washington University School of Medicine by Dr. Michael Schwartz of Denmark when he was in St. Louis on a fellowship. In this study, 110 recipients were used for passive transfer studies with ragweed serum. Equal numbers of the subjects were above and below the age of 50 years (up to age 96 ). The stronger concentrations of serum produced more wheal reactions in the skin of the older recipients than in the skin of recipients below age 50. However, with dilutions of serum, the skin of the younger persons showed positive reactions in the transfer test when the skin of the older persons failed to do so. The difference was attributed to a poor blood supply wasting away the $\mathrm{H}$-substance which accounts for whealing.

Lewis and Grant reported that a histamine wheal remained longer in the skin of subjects in the upper age group than in the skin of younger persons. Schwartz 
quotes Merrill Chase to the effect that a wheal caused by physiologic saline will persist longer in the skin of an older person than in the skin of a younger person. However, the diminution in erythema prevents more accurate interpretation of such observations.

Moderator Sheldon: Very good, Dr. Hampton. May I ask you this specific summarizing question? From the practical viewpoint, is it true that skin tests can be of diagnostic value in asthmatic and in allergic patients, even though the person is old and has senile changes in the skin?

Dr. Hampton: Yes-absolutely so.

Moderator Sheldon: Have you a comment, Dr. Rose, with relation to skin reactions?

Dr. Bram Rose: Not at this point.

Moderator Sheldon: Dr. Cooke, or Dr. Sherman?

Dr. Cooke: I have nothing else to say.

Moderator Sheldon: I want to call on Dr. Sherman, and ask him for the causes of asthma starting in middle age.

Dr. William B. Sherman: It is important to separate the causes of asthma into primary factors, which create the basic sensitization, and secondary factors, which precipitate attacks in a patient who already has primary asthma. The primary factors are well known-dust inhalation, foods, and occasionally drugs and infections - and may cause asthma at any age. As Dr. Cooke has already mentioned, multiple factors of different types may operate simultaneously in the same patient.

The secondary factors, which cause the asthmatic patient to have attacks at a particular time, include cold, damp weather, irritating odors and fumes, and exertion. Psychic influences are also important. Perhaps we shall discuss them later, but for the time being they can be classified as secondary factors which precipitate attacks in patients predisposed by certain primary factors. Infection must also be taken into account. In certain cases of asthma, infection may be the primary factor. However, in other patients, such as those who have asthma due to inhaled dusts or pollens, an acute respiratory infection can precipitate an asthmatic attack which is due fundamentally to the primary inhalation factor and only secondarily to the infection.

In the younger age group, from the age of 5 to the age of 40 years (omitting infants), probably 90 per cent of the cases of asthma are due to inhaled allergenspollens, dust, animal dander, feathers, and so on. At about age 40 , there is a rather abrupt reversal in the trend. Among new cases of asthma arising after the age of 40 , the intrinsic or infective type predominates. Only about 10 per cent of new cases of asthma arising after this age show positive results with skin tests for sensitivity to inhalants, pollens, and a few foods. The other 90 per cent are cases of so-called intrinsic asthma, chiefly representing allergy to infectionalthough I am not sure that all the members of the panel would agree. In any event, these are not simple cases of asthma in which one can elicit a specific reaction to some external factor and obtain therapeutic results by separating the patient from that factor. 
Along with this change in type of asthma with aging, there is a considerable increase in the incidence of asthma due to drugs. Among these drugs, aspirin is outstanding. It causes many more asthmatic reactions than all other such drugs combined. Thus, when we speak of drug asthma, we usually think in terms of aspirin sensitivity. Without doubt, these cases are much more common after age 40 than in younger groups.

I have nothing specific to say about endocrine factors. Perhaps Dr. Rose has some comment on that part of the problem.

Dr. Rose: I would list smoking as one of the secondary factors. I do not believe that smoking is a primary cause of the sensitization which produces asthma. However, there is no doubt that, in the patient susceptible to asthma, tobacco smoke is a secondary irritant that can precipitate attacks or make attacks worse. It probably is an important factor. There is evidence that smoking causes bronchitis rather than true bronchial asthma, although I would classify it as an important secondary factor in the latter.

Moderator Sheldon: Dr. Sherman, do you advise elderly patients with asthma to stop smoking?

Dr. Sherman: I often do, but if they show great distress, I do not always insist upon it. If I find anyone smoking more than a pack a day, I certainly advise him to cut down to reasonable limits. I find it very hard to get patients to stop completely. However, some of the enthusiastic ones who have stopped completely have had surprisingly great relief of their asthma.

Moderator Sheldon: I should like to ask the other members of the panel their reaction to smoking by persons beyond middle age. Dr. Cooke, what is your reaction?

Dr. Cooke: I think it is an important factor in asthma. There is a quantitative element in it. I would draw the line at about half a pack of cigarettes a day. We could almost limit the danger to the inhaling of cigarette smoke; it is more directly irritant than pipe smoking or even cigar smoking, with which there usually is not the same amount of inhalation of smoke. A pack of cigarettes a day can readily induce the congestion and the cough that will precipitate an asthmatic attack. Patients who indulge in excessive smoking, which I would say is above 6 cigarettes a day, have enough irritation to warrant advising them to reduce, if not to eliminate cigarettes. The problem of cutting down smoking is almost as difficult or worse than eliminating it. Smoking is a factor that must be taken into account.

Moderator Sheldon: Thank you, Dr. Cooke. Dr. Hampton, any comment? What do you do in this case?

Dr. Hampton: Let me comment on the remark that Dr. Sherman made about the 10 per cent of older persons with positive skin reactions. I am sure that Dr. Sherman means that 10 per cent will show positive skin reactions to the etiologic factors of the allergic disease. A larger percentage may show positive reactions, but these may be nonspecific and unrelated to the etiology.

Smoking plays a tremendous role. As Dr. Sherman stated, those who stop entirely are frequently quite surprised at the relief. 
In Boston, the statement has been made that particles the size of smoke are the etiologic factors in emphysema, whereas larger particles such as dust or pollen are etiologic factors in asthma. I try my best to induce the patient to stop smoking-although not the first time I see him. I try to gain his confidence, see him several times, admit that I am a smoker myself, and finally advise him to discontinue smoking.

Moderator Sheldon: Dr. Sherman, have you any rebuttal to Dr. Hampton's remarks with regard to the skin test?

Dr. Sherman: My figure of 10 per cent was intended to refer to actual causative factors proved by the results of treatment. Dr. Hampton might obtain a skin reaction in a higher percentage of cases, but $I$ doubt whether in more than 10 per cent one can show by treatment that asthma is due to the substance giving rise to the skin reaction.

Dr. Cooke: We ought to emphasize that what Dr. Sherman was talking about was cases of asthma that began after the age of 40 years, in which the percentage was not high. The carry-over from the younger group would make it considerably higher. In asthma that begins after age 40 , the element of sensitization factors as found in the younger group is much less. I definitely agree with him, but it should be emphasized that he was speaking of asthma that begins after age 40 , not that carried over from earlier life.

Moderator Sheldon: Dr. Rose, have you a comment about smoking?

Dr. Rose: I was going to say that, if the opinions of the panelists were inversely related to the square root of the volume of smoke that is going forth from this table at the moment, it would be quite clear that what they have said belies their actual thoughts, because no one gives a hoot whether he is smoking or not.

Moderator Sheldon: Don't do what I do, but what I say. Is that it? The question was brought up as to the relation of smoking to bronchitis. I should like to ask, How you differentiate true asthma and chronic bronchitis? Dr. Rose.

Dr. Rose: Differentiation between the extremes of the 2 conditions, namely, chronic obstructive pulmonary emphysema and bronchial asthma in the older patient, is relatively simple. With intermediate cases, the problem becomes somewhat more difficult.

Clinically, the history is first in importance. Asthma is characterized by paroxysmal attacks, which usually occur when the patient is at rest. In the person who has frequent attacks, they will be precipitated by emotion, exertion, cold air, and other secondary factors, as Dr. Sherman has mentioned. On the other hand, unless emphysema has progressed until the patient is breathless at rest, the dyspnea is usually related to some form of exertion or to an acute infection, so that it is important to find out what precipitates the attack of dyspnea.

The second point is that a person with emphysema will usually tell you that, when he is not too breathless, he can sleep in bed, lying flat and using 1 pillow. As the disease progresses, he may be forced to use more than 1 or 2 pillows, but this does not happen as frequently as in the person with asthma. With the onset of asthma and true bronchial wheezing - I call it bronchial spasm; Dr. Cooke 
likes to call it bronchial obstruction - the victim usually requires 2 , and sometimes 3 pillows, even though there may be no cardiac involvement. This is not a crucial differentiating factor, but it is helpful at times.

The family history must be taken into consideration. Most persons with true obstructive pulmonary emphysema have no family history of allergy, whereas a fair proportion of those with asthma do.

A person with advanced emphysema usually has the characteristic barrel chest with an increased antero-posterior diameter, and a flattened diaphragm. To say that this is not seen in cases of asthma is not true, but barreling is far less apparent in the latter condition. The emphysema patient, during moderate to acute attacks, is often cyanotic. The asthma patient is rarely cyanotic unless there is acute status asthmaticus.

In cases of emphysema, there is frequently evidence of right-sided cardiac involvement with increased venous distention. I do not believe, although some members of this panel will disagree with me, that asthma leads to emphysema. The heart sounds are not of great diagnostic importance, but in the patient with emphysema the second pulmonary sound will often be more accentuated than in the normal person. This is rarely observed in the patient with asthma.

The degree of relief obtained with different types of medication also helps to establish the nature of the attack.

A few biochemical changes occur in emphysema that are not commonly found in asthma, namely, an elevated $\mathrm{CO}_{2}$ content and moderate lowering of the $\mathrm{pH}$ of the blood. This is not a great help in evaluating an office case clinically, but it is important with regard to the type of treatment. This is particularly true when determining the amount of oxygen that should be given to a patient admitted to the hospital with acute dyspnea, in whom the differentiation between asthma and emphysema is uncertain.

Finally, there is a point with reference to the sputum. If the patient has asthma, at some time or other the sputum may contain mucoid spirals. These are, so far as I know, never found in emphysema. Beyond that, I would not like to go. Even if one uses complicated pulmonary function tests, there is little to differentiate the patient with obstructive pulmonary emphysema from the patient with reversible emphysema associated with an acute prolonged attack of asthma.

Moderator Sheldon: Thank you, very much. Would any other member of the panel care to comment?

Dr. Hampton: I want to challenge Dr. Rose's statement that asthma does not lead to pulmonary emphysema. The high incidence of pulmonary emphysema in what is known as infective or intrinsic asthma, furnishes statistical evidence that the cough of asthma leads to emphysema. Whether or not asthma leads to emphysema, the cough of asthma leads to emphysema, just as the cough of tuberculosis and the cough of chronic bronchitis lead to it. Pulmonary emphysema may also be seen in the younger age groups, even in children and in those of teen age who have had asthma since early life.

I know that it does not come within the scope of this discussion to enlarge 
upon the reasoning as to whether or not asthma leads to emphysema, but I should like to challenge Dr. Rose on this statement. No doubt he has some well founded investigative work to substantiate it. To me, it is always amazing to see the inconsistency with which pulmonary emphysema develops in the asthmatic patient. There seems to be some sort of predilection. A person may have asthma for a long time and yet not have pulmonary emphysema, whereas a person who has had asthma for a shorter time may soon suffer from intractable and severe pulmonary emphysema in addition.

Moderator Sheldon: Dr. Cooke, any comment?

Dr. Cooks: I think that blocking caused by intrabronchial obstruction is an important factor in producing distention of the lung, which is really what emphysema is. There is so much evidence of it in the asthmatic patient that we must consider the relationship between asthma and the obstruction. Either difficult breathing or coughing tends to promote distention of the alveoli and, therefore, the development of that condition in the lung.

Moderator Sheldon: Dr. Rose, I should like to give you time for rebuttal.

DR. Rose: Thank you, Dr. Sheldon. We have been interested in the problem of the cause of obstructive pulmonary emphysema, particularly with relation to bronchospasm. A number of years ago, we studied such a group by means of cardiac catheterization. We chose persons who looked as though they should have emphysema, whose diaphragms were low, and whose lungs were hyperaerated during attacks. We catheterized their hearts. In contradistinction to persons with true obstructive pulmonary emphysema, there was no pulmonary hypertension. When a moderate degree was induced, it was purely secondary to intrapulmonary changes, that is, changes within the thorax itself.

In asthma, during the expiratory phase the blood pressure is elevated, and during the inspiratory phase it falls to normal. This is not true of emphysema. I could continue at great length, but in view of the friendship that exists between me and my friends at this table, with whom I heartily disagree, I shall desist.

Moderator Sheldon: Thank you, Dr. Rose. I should like to have Dr. Cooke comment about asthma and its relation to the production of emphysema, being a bit more inclusive.

Dr. Cooke: It seems to me that this has developed into a question as to what is emphysema, and what is not.

The general consensus is that the term emphysema means distention of the alveoli throughout the lungs. It seems paradoxical to talk about obstructive emphysema when obstruction is exactly what exists in the asthmatic patient. The basis for asthma is an obstruction in the bronchi, caused largely by thickenint of the bronchial wall and by the very curious and tenacious mucoid plugs. There may also be an element of bronchial spasm. I do not agree entirely that asthma and bronchospasm are synonomous, because there is little evidence for a greater degree of spasm in emphysema than in asthma, or vice versa.

The degree of bronchial obstruction depends entirely upon the age of the patient and the duration of the asthma. We see children who are particularly resistant to therapy and have prolonged asthmatic attacks, defying all present 
medical procedures. Children may have asthma at age 8 or even earlier, and be continuously asthmatic for years. All the evidences of asthmatic obstruction may develop, including the barrel chest and even a moderate degree of rightsided cardiac enlargement. The point is that from time to time these children have not only their chronic asthmatic condition, but more. In those who have short and periodic attacks of asthma, dilatation of the alveoli occurs to some extent. If they are relieved, the process is reversible. It is reversible because the tissues are young and have retained their elasticity. In the older age group, however, tissues have begun to undergo change. We speak of it as atrophy. It affects not only the lung, but the skin, the muscles and all other tissues, so that there is loss of vitality and elasticity. In cases of persistent and prolonged dyspnea due to asthma, we see this marked type of distention, i.e., emphysema by roentgenographic and clinical evidence has developed into an important factor. The elderly have passed the age when emphysema will disappear, even if the asthmatic factor is relieved. The tissues have lost the capacity to return to their normal or original state.

In addition to emphysema in these cases, there is an altogether different process, namely, pulmonary fibrosis. The evidence of fibrosis during life is chiefly indirect-in roentgenograms of the chest. Fibrosis is difficult to demonstrate directly, other than at necropsy. The marked increase in pulmonary shadows, the accentuation of the bronchial markings, and other changes throughout the lung are seen in these prolonged cases. The fibrosis is secondary to infection. That is one of the reasons why we see more of it in older people than in children. However, children with prolonged asthmatic conditions resistant to medication may have not only emphysema but also pulmonary fibrosis. In such cases, there is always evidence of infection, whether primary or secondary.

The development of fibrosis is really the end-result of infection in the bronchi and glands; it is possibly secondary to sinus involvement, which accentuates it. Fibrosis is a part of the picture induced not only by obstruction (emphysema) but by the infection which is an important feature in prolonged cases. When there is obstruction in the bronchial tree, there must be dilatation of the alveoli as a result of the dyspneic respiratory effort and coughing. This is my concept of emphysema. Emphysema is reversible when the attacks are occasional or periodic as in youth, and irreversible when the condition is prolonged.

Moderator Sheldon: Thank you, Dr. Cooke.

I should like to ask Dr. Sherman a question, which I hope will define what we are coming to in relation to the management of asthma. What things, other than those already discussed, would you have in mind when you see a patient who is short of breath and wheezing?

Dr. Sherman: In dealing with middle-aged patients, more care must be exercised in making the diagnosis of asthma. In children and young adults, bronchial asthma is by far the most common cause of wheezing and periodic dyspnea. In older people, one must also take into account the possibility of organic obstructions, of tumors either inside the bronchi or pressing on the bronchi, of pulmonary fibrosis due to silicosis or other pulmonary disease, and a 
great many other factors. How does one differentiate these? It can be done by resisting the tendency to conclude that the patient has bronchial asthma, by taking a careful history, by performing a careful physical examination (noting particularly if the signs and symptoms are localized in one lung or one part of a lung), and by examining the sputum for the features characteristic of asthma. For instance, if the sputum is bloody, there is reason to suspect a lesion inside the bronchi. Perhaps most important is roentgenographic examination of the chest. Obviously, every patient with asthma should have a roentgenogram of the chest as a part of his examination. It becomes even more important in dealing with middle-aged and elderly patients. Most of the lesions to which I have referred can be recognized by means of careful roentgenographic examination of the chest.

A test which may help to differentiate organic obstructions from asthma, is the effect of epinephrine upon the wheezing. If epinephrine produces rapid and striking relief, the more likely diagnosis is asthma. If epinephrine does not produce relief, one has to think more seriously of other conditions. Bronchoscopy is a valuable procedure when in doubt; only thus can some of these problems be settled.

Another aspect is the differentiation of cardiac asthma or cardiac dystonia from bronchial asthma. Again, this is much more difficult in elderly patients than in young patients. In the latter, asthma is usually due to extrinsic factors. It is likely to be acute, periodic, and of the bronchial type. Heart disease in young patients is usually valvular, and relatively easy to diagnose if the heart is carefully examined. In older patients, asthma is not so characteristically periodic It tends to be of the chronic, low-grade variety. Heart disease in older patents is more often of the arterial type. There are no characteristic murmurs, so it is not as easily recognized. It is important to remember that both arteriosclerotic heart disease and asthma are exceedingly common in this older age group. Therefore, the patient frequently has both conditions. It becomes not so much a question of differential diagnosis as of studying the patient carefully from both aspects and not jumping to the conclusion that, because he has arteriosclerotic heart disease, he does not have bronchial asthma as well.

We shall not have time to discuss the different methods for diagnosis of arteriosclerotic heart disease. It should be emphasized, however, that in the older age group, it is common to have asthma and heart disease in the same patient. These diseases must be treated simultaneously, but separately.

Moderator Sheldon: Thank you, Dr. Sherman. Does any other member of the panel wish to comment?

Dr. Sherman told us of bronchoscopy as a diagnostic procedure. I should like to comment in a positive way relative to bronchoscopy and the instillation of contrast media into the bronchus, and see if the panel agrees or disagrees with me. To my way of thinking, these procedures offer a valuable diagnostic aid, but therapeutically they are disappointing. I suspect that the instillation of oils does harm rather than good. Does anyone on the panel disagree with me?

Let us proceed, then, to the therapy of elderly persons with asthma. We 
try to define it by indirection. I shall call upon Dr. Cooke first, and ask him how he would manage an elderly patient with bronchial asthma.

Dr. Cooke: Dr. Sheldon asked this question as though the diagnosis of asthma had been made. When these patients present themselves, and before we start to do anything in the way of management, we have to make a diagnosis. Perhaps the patient has been referred with a history of asthmatic attacks. It is our function to decide whether the disease is asthma or not. That has just been discussed by Dr. Sherman.

The next step is to ask ourselves: "If this is an asthmatic condition characteristic of the middle-aged or elderly, what is the cause?" One cannot manage any patient properly and satisfactorily without a clear-cut conception of the cause of the difficulty. Therefore, a careful study must first be made. This involves the past history, particularly with regard to familial allergic disease. Has the patient inherited an allergic constitution? Has he at any time exhibited evidence of sensitization to anything, or has he suffered from some disturbance such as drug sensitivity, that is not a factor in the present condition. Such a patient will probably be found to have the capacity to become allergic to certain things, such as drugs. For example, in many patients who are sensitive to aspirin, asthma is often the chief, and sometimes the sole, evidence of their sensitization.

How did the condition develop? Was the onset rapid or gradual, and what were the circumstances? Was he at home? Environmental factors are always important in these cases, in addition to certain other intrinsic factors.

After a careful history, it is necessary to make a careful and full physical examination. Once this is done, and you have concluded that the disease is asthma, it is your task to carry out the various procedures that will not only confirm this conclusion but contribute toward determining the cause. The status of the heart and the kidneys, and the general physical condition must be determined. Laboratory tests of various sorts should be made. Roentgenographic examination in asthmatic patients of this age group should include the sinuses. The possibility of dental infection should receive particular attention. For instance, if there is infection in the upper molar areas, it is likely to have spread to the maxillary antrum, and the antrum infection may clear up after the removal of the diseased tooth.

Having made these studies, skin tests should be undertaken in all cases, because they help to throw light upon certain factors. Not all positive results are significant, so careful clinical correlation is important. It is of no particular moment that a man with chronic asthma is allergic to some food that he eats only once or twice a year (for example, mushrooms). A positive reaction to, or a history of being sensitive to, mushrooms does not solve his immediate problem. He has a chronic condition. It takes continuous contact with a causative factor to maintain his allergic manifestations. Having determined the possible causes by means of careful clinical observation, the physician may remove the patient from his environment and place him where he does not have to come in contact with the offending allergen, for example, the dust in the factory, office, or home. 
If the patient is symptom-free in one place but not in another, the external environmental factors can be sought. Though careful clinical observations and trials must be made in order to satisfy the requirements, that does not mean the performance of the hundreds of available skin tests. A clear-cut idea of the nature of the trouble can be obtained by a screening method, involving perhaps half a dozen of the more important inhalant allergens. Foods can be covered by 10 or 12 food tests.

It is characteristic of allergic disease that rarely is there a single cause. Multiple sensitization is the rule rather than the exception, and persons who have an allergic disease are likely to be sensitive to 3 or 4 things. Infection should not be overlooked. I hope Dr. Sheldon will bring it up as a factor, and mention what may be done about it.

One must decide whether or not a respiratory-tract infection is present, and whether or not there has been removal of nasal polypi. Has there ever been evidence of true sinusitis? These conditions, confirmed by roentgenographic examination, are important factors in preparation for management. The patient is treated symptomatically while we are making serious efforts to discover the cause. Without a fair conception of the cause, one cannot manage these cases properly.

Moderator Sheldon: Thank you, Dr. Cooke. We shall come back to the question of the management of infection. I should like, now, to ask various members of the panel what specific medications may be used in the asthmatic patient, not only during the acute attack, but in the prevention of future attacks.

First, I am going to ask Dr. Hampton whether or not iodides are of any value in the treatment of asthma, and when they are indicated.

Dr. Hampton: Expectorants are important agents in symptomatic therapy for the treatment even of mild cases of asthma and emphysema. In the patient with severe asthma, expectorants probably head the list, as far as symptomatic therapy is concerned. An expectorant is just as valuable, if not more so, as the so-called bronchodilators and agents used to reduce bronchial edema, such as ACTH and steroids. The literature contains many necropsy reports showing that death from asthma is due to anoxia resulting from extensive bronchial mucus. Therefore, expectorants are of prime importance, particularly in the patient acutely ill with asthma.

Iodides are of great value as expectorants, that is, potassium iodide by mouth and sodium iodide intravenously. Time does permit a discussion of the pros and cons of the pharmacologic effect of iodides. There is some evidence that the action is not directly on the bronchi, but a reflex pathway in the stomach; if so, this would rule out intravenous iodides as effective agents. Other investigators, however, have obtained contrary results. When the patient is extremely ill, intravenous iodides are very effective in addition to oral iodides in large doses. Iodides should not be given intravenously until they have first been given orally in order to determine possible sensitivity.

Lobelia, an old herb now almost discarded, is an effective expectorant. Then 
I would list ammonium chloride, glycyrrhiza, and glycerine. Thus, there are other expectorants to substitute for iodides, if the patient should prove to be iodide sensitive.

In treating acute attacks of asthma, intravenous iodides will blend with other preparations, such as ACTH and theophylline. In St. Louis, we call this combination an intravenous cocktail. Iodides should be forced, as they dissolve the sticky mucus which can cause death in cases of asthma.

Moderator Sheldon: Does any member of the panel disagree with Dr. Hampton, or wish to add anything with regard to expectorants? If not, I should like to consider the next subject-the so-called bronchial dilators, and ask Dr. Sherman to speak about them.

Dr. Sherman: Bronchial dilators are, in a sense, the "backbone" of the treatment of bronchial asthma, which is primarily a matter of obstruction of the smaller bronchi and bronchioles.

Two groups of drugs are useful in this regard-the epinephrine group and the xanthines, among which aminophylline is the primary drug. The epinephrine group includes adrenaline and many related products. The most important ones are epinephrine itself, and ephedrine for the immediate treatment of the acute asthmatic attack. Epinephrine is still the first drug to try, and usually the most effective. It has the disadvantage that it is not potent when taken by mouth, so that it is less useful for treatment of the patient at home. However, it is effective by inhalation as an aerosol. There are many types of epinephrine products for inhalation, all of which are essentially similar and valuable in relieving mild attacks of asthma.

For oral treatment, ephedrine is the primary agent and probably does everything that any of the other drugs of the group will do. It provides effective relief for relatively mild asthma, and can be used in the home because it is taken orally.

Ephedrine may be combined with other drugs, such as mild sedatives. As ephedrine is somewhat stimulating, these combinations are more practical and effective than ephedrine alone. It is easy to adjust the amount of ephedrine and of sedative in capsule form for a particular patient so that he will not be stimulated by the drug to the point of interference with sleep, but neither will he have so much sedative as to become sleepy in the daytime.

Of the other drugs in this group, we need mention only one, isopropylarterenol, which is more familiar under the trade names of Isuprel and Norisodrine. It is as effective as epinephrine when administered by aerosol inhalation or inhaled as a powder.

Among the xanthines, aminophylline is valuable and is the most commonly used. If epinephrine does not relieve the acute attack, the next drug to try is aminophylline, intravenously. It is so potent that it must be injected very slowly, taking five or ten minutes to administer the whole intravenous dose. If the patient is in the hospital, it is often preferable to dilute the dose with 100 or 200 cc. of dextrose and give it by intravenous drip, in order to control the rate of administration. 
By mouth, aminophylline and the other xanthines are irritating and likely to cause nausea and vomiting when given in doses large enough to control severe asthma. They are most useful in combination with ephedrine and sedatives.

Aminophylline is effective by rectum, and therefore is useful for home treatment of the patient who has an attack not easily relieved by aerosol inhalation or by ephedrine orally. Aminophylline may be given in a rectal suppository or dissolved in a retention enema. It provides effective and practical home treatment.

Moderator Sheldon: Thank you, Dr. Sherman. What dose of epinephrine is indicated in an asthmatic patient?

Dr. Sherman: That is a very good point. The dose is exceedingly variable. For that reason I would suggest a modest dose initially, perhaps 0.4 cc. of 1:1000 solution. One should wait five or ten minutes to see if the patient obtains relief. There should be no hesitation in repeating the same dose in five or ten minutes, if necessary. It is so hard to judge the correct dose, that it is much better to give a small dose (with the idea that a second one may be necessary in ten minutes) than to attempt to guess the total requirement at first.

When we give what I call a relatively large dose-1 cc. of 1:1000 solution-it does less good than it should, because the local constricting effect of epinephrine interferes with its absorption. Two small doses have a better effect than one large one.

Moderator Sheldon: Are there any contraindications to the use of epinephrine in this case?

Dr. Sherman: I can think of nothing that would stop me from trying epinephrine in a patient having a severe, acute asthmatic attack. Even if the patient were hypertensive or known to have heart disease, I would not hesitate to give him a slightly reduced dose- 0.3 cc. of $1: 1000$ solution-watching him carefully, and repeating the dose in five or ten minutes.

We may worry about the presence of hypertension and heart disease, but asthma has such an adverse effect upon both of these conditions that, when epinephrine relieves the asthma, we have helped the patient a great deal more by giving this drug than by worrying about the contraindications.

Moderator Sheldon: I agree with you. There might be one rare instance-I can hardly visualize it being a problem that will confront anyone-wherein fear of giving epinephrine might arise. That is in the person receiving chloroform anesthesia. However, if the patient is under anesthesia, one is not going to be worried about asthma anyway.

Does any member of the panel disagree with this statement? It is important to remember that there really are no primary contraindications to the administration of epinephrine.

Dr. Cooke: Might it not be well to emphasize the fact that, when a patient with hypertension is given a dose of epinephrine and the blood pressure reading is taken again in ten or fifteen minutes, it will have dropped rather than increased as a result of the epinephrine. The effort to breathe increases the pressure and is a large factor in producing the degree of tension that one finds 
at the instant. In a patient with severe dyspnea the blood pressure may be increased; the relief obtained rapidly with epinephrine induces lowering.

Moderator Sheldon: Thank you, Dr. Cooke.

Now, I should like to start a discussion on some compounds that are tremendously interesting and valuable in the treatment of middle-aged and elderly asthmatic patients. I shall call upon Dr. Rose to give us his views on the use and abuse of, and indications and contraindications for, corticotropin and corticosteroids.

DR. Rose: This group of compounds undoubtedly constitutes one of the most useful groups of drugs for the treatment of asthma, as well as other conditions in the older patient.

The question is often asked: "Which substance should one use?" "Would you use corticotropin in preference to cortisone or the other steroids? If so, why?" The first question that one has to answer is, "Are there any real contraindications?"

The first consideration has to do with the status of the patient. Has he been bedridden? If so, how much osteoporosis does he have? Fortunately, among asthmatics the incidence of osteoporosis with spontaneous fracture of a vertebra is much lower than in patients with arthritis, for example. I have seen it in only 1 case of asthma.

Secondly, "Is diabetes present? If so, is it a contraindication to corticosteroid therapy?" In my experience, it is not.

A history of mental depression is a factor to be considered. I know that these points have been belabored many times, but they are important. So are the questions, "Does the patient have any tendency toward heartburn? Is there a history of ulcer? Is cardiac failure present? If so, does that modify your choice of compound?"

I think there are no significant contraindications to corticosteroid therapy so long as one is aware of the possible side-effects and adopts methods of preventing their occurrence. Corticosteroids are best used in self-limiting diseases, such as seasonal hay fever or asthma, when one knows that they will be given for only a short time.

Does the adrenal of the older person respond as rapidly and as well to a dose of corticotropin as does that of the young, robust person? We know from studies on steroid excretion that both urinary 17-ketosteroids and glucocorticoids are reduced to some extent in elderly persons. It is not known whether this change is controlled by the adrenal or by the pituitary gland.

It does not much matter which corticosteroid you use, as long as you give an adequate dose. Because of the ease of administering the oral corticosteroids, we use them in preference to preparations of ACTH. There is also the possibility that ACTH, given in a dose of 20 units by intravenous drip, may induce sensitization.

With reference to the excretion of adrenal steroids in the urine, one additional feature might be mentioned. We have found that in most older asthmatic pa- 
tients the level of steroid excretion is considerably lower than in nonasthmatic patients of the same age. Apparently the adrenal is less capable of responding to ACTH than in normal persons.

The most recent oral steroids are by far the best-prednisone and prednisolone. They do not affect the electrolyte balance nearly so much as the older compounds. Dependent edema does not develop. When there is any possibility of the presence of a cardiac disorder, these are the steroids of choice.

We like to start with a sufficient dosage, depending upon the severity of the illness, and then decrease it to a maintenance level. The maintenance dosage will vary considerably. In some older persons, it will be at the surprisingly low level of $5 \mathrm{mg}$. of prednisone once a day, and in some instances, once every second day. Higher dosages are sometimes indicated. It is better to start at a high level and lower it than to start at a low level and wonder when a remission will commence. Failure to respond may be due to intercurrent infection. This must always be kept in mind.

If a patient has been maintained with prednisone, what is the advantage of giving him adrenocorticotropin as a booster, in the form of one of the new zinc preparations? In my experience there is none. If you give prednisone, both the adrenal and the pituitary will be suppressed. Then one wonders whether or not something should be done to maintain the integrity of the adrenal cortex. Why not give 2 or 3 injections of corticotropin (ACTH)? The reason for not doing so is this: Prednisone suppresses the adrenal cortex for a much longer period of time than following administration of other oral steroids including hydrocortisone and cortisone-even as long as six months. Thus, when a person, who has received prednisone and then been maintained by other means, is given corticotropin within one to three months after prednisone has been discontinued, the adrenal probably cannot be stimulated. A longer interval after omission of prednisone is required. Even if one succeeds in stimulating the adrenal, the injections of ACTH depress the pituitary further. Oral adrenal steroid therapy is reinstated, and before long one is back to the starting point. What good will have been done? As far as I can see, none, except to have satisfied yourself that you have done what is said to be the best thing. Because I do not know whether it is the best thing or not, I do not resort to so-called booster injections of corticotropin at intervals of six months, a year, or any other time.

Other standard types of therapy should be used in controlling asthma when the patient is receiving prednisone or some other steroid. By so doing, the amount of steroid necessary will be decreased, the likelihood of ultimate discontinuation of steroids will be increased, and the patient will save money.

Moderator Sheldon: On the question I asked, you could spend a full hour and not cover the subject completely. I should like to clarify a few things, if I may, Dr. Rose. You mentioned that, initially, patients should receive a relatively high dosage of a corticosteroid. You stated that your choice might be prednisone. Assuming that the patient is elderly, what do you mean by high dosage?

Dr. Rose: It depends upon the status of the patient. For example, if asthma 
of a troublesome degree has not responded to bronchial dilators or expectorants, 20 to $40 \mathrm{mg}$. of prednisone daily for two days will probably prove adequate. The dosage can soon be reduced.

If a patient with acute asthma receives initially a daily dosage of 20 or 30 mg., he will not respond for five or six days. On the other hand, if he is given $\mathbf{8 0}$ mg. in fractional doses the first day, and $60 \mathrm{mg}$. the second day, he will improve within thirty-six to forty-eight hours. The dosage can then be reduced rapidly to a level commensurate with the severity of the disease. This plan saves time for the patient, and time and worry for the physician.

Moderator Sheldon: Thank you. Now I should like to ask you 2 more questions.

What would persuade you to administer a corticosteroid to an asthmatic patient? Obviously, not all asthmatic patients receive steroids initially.

Dr. Rose: Dr. Sheldon, I assume that everything else has been tried. Let us suppose that the patient has been to you, and has not done well. Then he has gone to Dr. Sherman, or Dr. Hampton, and he has done a bit better, but not as well as he ought. Finally, he has been seen by Dr. Cooke, who has tried all possible therapeutic measures. I am sure that Dr. Cooke would treat such a patient with steroids.

I start steroid therapy only when a patient is acutely ill and requires immediate relief not obtainable by other means, or when the patient has been a chronic invalid for a number of years and has not responded to conventional forms of therapy. This point should be emphasized-as we have learned from our experience during the past six years, when we have had ample opportunity to study these patients both in the wards and in the clinic. The whole aspect of the clinic has changed. Prior to the advent of steroids, we had a large indigent clientele. Many were unable to work, and unable to support themselves. Our therapy, obviously, was not good enough. Now, many of them receive steroids in low dosages, under continual watchful supervision. We are vigilant concerning the onset of such things as tuberculosis and other infections, or whatever may arise. Many of the patients are employed in the hospital. Those who are not, are able to take a job outside and support their families. They can afford to pay for their medication, whereas, previously, they could not.

Moderator Sheldon: Would you elect to use corticosteroids in case of status asthmaticus of several days' duration in a patient who had not received corticotropin or corticosteroids before you saw him?

DR. Rose: Yes, I certainly would. I can see no contraindication.

Moderator Sheldon: Would you use corticosteroids in preference to intravenous corticotropin?

DR. Rose: That would depend largely upon whether or not the patient had recently been receiving oral steroids. If the patient had had corticotropin previously and a rapid result was desired, ACTH could be given by slow intravenous drip along with intramuscular injections of the long-acting zinc preparation in a dosage or 20 to 30 units twice daily until a response was obtained. On the other hand, if the patient had, as recently as one month or six weeks previously, 
been receiving an oral steroid for a considerable length of time, the treatment of choice would be high doses of corticosteroid orally.

Moderator Sheldon: How often do you think that a patient receiving chronic corticosteroid therapy should be seen by his physician?

Dr. Rose: The plan that we follow is this: Patients receiving routine therapy (that is, a maintenance dosage of 5 or $10 \mathrm{mg}$. of Meticorten per day) report by telephone, or come to the clinic or the office at least once every two weeks. They do not have to come as long as they feel well and as long as nothing bothers them. We insist upon seeing them regularly each year at intervals of five to six months in order to re-evaluate their status, and to make sure that no unwanted side-effects are developing.

Moderator Sheldon: You have alluded to the various complications that may follow prolonged corticosteroid therapy. What have you observed that was troublesome? What are some of the common complications of prolonged corticosteroid therapy?

Dr. Rose: The majority of patients have little trouble. Some, however, become susceptible to infection. This can be troublesome and must be dealt with appropriately.

Three patients in our series, which is a large one, have osteoporosis with spontaneous fractures. Two of them have the rare combination of asthma and advanced arthritis.

Other than that, Dr. Sheldon, I would say that there have been no serious consequences. We have had only 1 case of perforated ulcer, and that was in a patient with periarteritis nodosa. There have been some cases of gastric distress, which was readily controlled with an antacid or an anticholinergic drug along with an appropriate diet.

Acute collapse may occur in the presence of infection in a patient who has recently stopped steroid medication. This is due in part to suppression of the pituitary-adrenal mechanism, and it requires rapid and heroic measures.

Moderator Sheldon: Do you keep your patients under the so-called Albright regimen, if they are receiving corticosteroids for a prolonged period?

DR. Rose: It depends largely upon the type of person. If he is robust and active, one generally has little to fear with reference to the development of osteoporosis. It is only in sedentary or bedridden persons that this need be considered. Even then, I do not know whether Albright's regimen will prevent marked osteoporosis. We do not know just what metabolic mechanism is involved in this loss of calcium and protein which results in decalcification of bone.

We add testosterone to the treatment program, particularly in the older age group, if we think it is indicated. To some patients, we give supplementary calcium.

Moderator Sheldon: Dr. Rose, are you concerned if your patient comes to operation after prolonged corticosteroid therapy? If so, do you take any precautions with regard to surgical procedures?

DR. Rose: Any patient, who receives steroid therapy for any length of time, is warned that he must always tell any physician he may consult that he has been 
taking steroids. We have been able to follow those of our patients who have undergone operations-for instance, thyroidectomy, appendectomy, or even an abdominal perineal resection, which is an extensive procedure. We have lost only 1 patient among 15 or 20 undergoing a major operation. ${ }^{1}$

Moderator Sheldon: Thank you very much. We could go on and on, but I should like to give the other members of the panel an opportunity either to agree or disagree with Dr. Rose and to add any comments of importance that they care to make.

First I shall call on Dr. Cooke.

Dr. Cooke: I am sure that Dr. Rose mentioned this. We should always attempt, in patient receiving corticosteroids for asthma, to deal with the causative factor. We should not see patients, make a diagnosis of asthma, and resort to this type of therapy for their immediate relief without at the same time making a serious effort to find the cause. If there is infection, continuous antibiotic therapy or chemotherapy should be instituted. The patient may belong to a group for whom nothing else can be done, but we must always try to find the causative factor and treat it at the same time that the patient receives continuous therapy for relief of symptoms.

Moderator Sheldon: Thank you, Dr. Cooke. Dr. Sherman?

Dr. Sherman: I think that the subject has been covered.

Moderator Sheldon: Dr. Hampton?

Dr. Hampton: In our study in St. Louis, continuous infusions of ACTH for twenty-four to ninety-six hours resulted in a much greater excretion of 17ketosteroids than intermittent eight-hour daily infusions of the same dose of ACTH. Moreover, although a single injection of 40 units of ACTH-gel led to very little increase in the 24-hour excretion of 17-ketosteroids, the excretion after four days of ACTH-gel approached that associated with daily eight-hour infusions of regular ACTH. The newer corticosteroids, such as Meticorten, approach the clinical effectiveness of ACTH-gel.

I should like to ask Dr. Rose 2 questions. He stated that steroids used for maintenance therapy may depress the activity of the adrenal cortex for periods up to six months. He also mentioned that ACTH suppresses the pituitary. Will the suppressive effect of ACTH last as long as that of steroids? What is your experience when comparing prednisone with prednisolone? We have found that prednisone and prednisolone are equivalent in clinical effect, although there are situations in which prednisone may be slightly more effective than prednisolone, and vice versa.

Dr. Rose: I cannot answer the first question, and I know of no work that would answer it reasonably. I agree with Dr. Hampton that, clinically, there is no difference between the therapeutic effects of prednisone and prednisolone.

Moderator Sheldon: Thank you very much.

We have much material to cover and want to allow a few minutes at the end

${ }_{1}$ Our routine procedure is to give $200 \mathrm{mg}$. of cortisone or equivalent per twenty-four hours in fractional doses on the day before, the day of, and the day following operation, after which the dose is slowly reduced. 
for audience participation. Therefore, I am going to ask several questions and request that they be answered briefly. Dr. Hampton, will you please speak of the use of oxygen and aerosols.

Dr. Hampton: Although oxygen is necessary for life, it is a two-edged sword. The administration of 100 per cent oxygen by mask, or even by intranasal tube, may be dangerous. In a case of severe asthma, the physician should stand by and watch the patient for signs of carbon dioxide narcosis, at least when oxygen therapy is initiated.

Aerosols have their place. They serve as sputum solvents and bronchodilators, particularly in combination with intermittent positive pressure breathing as supplied by the Bennett and the Emerson IPPB respirators. There is controversy over the matter of whether a bronchodilator substance, such as epinephrine or isopropylarterenol, may be just as effective when administered as an ordinary aerosol as by intermittent pressure breathing. Motley (who introduced IPPB), Segal and his associates, Smart and his co-workers, and others including ourselves have found that 100 per cent oxygen can be administered with intermittent pressure breathing without increasing $\mathrm{CO}_{2}$ narcosis. A practical bronchodilator-sputum solvent for use in the nebulizer of the intermittent pressure apparatus would be $0.5 \mathrm{cc}$. of isopropylarterenol $1: 200$ diluted with $5 \mathrm{cc}$. of a detergent such as Alevaire or Tergimist.

The same materials may be given as ordinary aerosols by using compressed air as the force. Even pressure produced by a bicycle pump will do. A hand nebulizer may be used for spot relief. The latter has its place, provided the patient does not use it every fifteen minutes or so when he is obviously refractory to its effect because of the mucus in the smaller bronchi.

Moderator SHeldon: Thank you. Do you agree with the statement that, to date, enzymes such as trypsin are hazardous to use as aerosols in elderly asthmatic patients?

Dr. Hampton: They may be hazardous. Crystalline trypsin (Tryptar) may be used in selected cases, but it is highly irritating and should not be used in the concentration ordinarily advised (125,000 units). Concentrations of 50,000 units diluted in 3 cc. of diluent, given every six to twelve hours for a limited number of treatments ( 3 to 6 ) is a safer dosage schedule. Desoxyribonuclease (pancreatic dornase) is less irritating and, like trypsin, may be a useful therapeutic tool, particularly when the sputum is purulent.

Moderator Sheldon: I should like to know whether you agree or disagree on one other question. Is it not true that oxygen is a potent anti-expectorant, and that in its use one risks promoting further bronchial plugging?

Dr. Hampton: I pass.

Moderator Sheldon: Has anyone else a comment to make concerning oxygen or aerosols?

If not, let me ask Dr. Sherman to give his view as to the use of antihistamines in asthmatic patients.

Dr. Sherman: Antihistamines are vastly overrated as treatment for asthma. Many asthmatic patients who have been treated by general practitioners rely 
on an antihistamine as their main treatment. Often, they do not obtain worthwhile results. A few mild cases of asthma may be relieved by antihistamines, but only the mildest cases, and even these would be better relieved by oral ephedrine or aminophylline. The only indication for the use of an antihistamine is in the patient who is made uncomfortable by the side-effects of ephedrine. I would always combine the antihistamine with aminophylline in order to obtain the additional benefit of aminophylline. I would not rely on an antihistamine as the primary drug to relieve acute asthma.

Moderator Sheldon: Does the panel agree? I should like to proceed to the next point, which I think is important, and that is, the use of fuids in hospital patients. Dr. Cooke.

Dr. CoOkE: This would apply to cases of severe asthma. At the time of hospital admission many patients have had severe dyspnea for a number of days. They are greatly dehydrated. The mere act of breathing eliminates a great deal of water. Under such conditions, the patient may not want to take fluids by mouth. Therefore, it is important to treat these patients with intravenous fluids, on an emergency basis. This provides an opportunity to give not only corticotropin, but aminophylline and glucose as well.

The use of intravenous fluids in severe cases of asthma is an important part of treatment, particularly when there is an element of dehydration. Dehydration tends to increase inspissation of the bronchial plugs, so that breathing becomes more difficult.

In severe cases, it is important to pay some attention to diet and supplementary medications. Many patients suffer from abdominal distention. They may have taken drugs to stop coughing, and have become severely constipated. The bowels may not move for several days, resulting in distention with pressure on the diaphragm, which adds to the asthmatic difficulties. Therefore, when these patients are first admitted it is our practice to give them nothing but fluids for a day or two-by mouth, as soon as possible. Proper attention is also given to clearing the bowel with cathartics, with colonic irrigations, or with enemas in order to lessen intra-abdominal pressure. This is important.

Moderator Sheldon: Thank you, Dr. Cooke.

I should like to ask Dr. Hampton to give briefly his experience and state whether tranquilizers and sedatives are useful in asthma.

Dr. Hampton: Briefly, yes. Sedation is important in asthma. The patient has anxiety because he cannot get his breath. Actually, he cannot get his breath out, but he thinks he cannot get it in. For years, barbiturates and other sedatives have been used.

The new tranquilizing drugs, according to my short experience, have a definite place as adjuncts in the management of asthma in the aged as well as in younger persons. The hospitalized patient with severe acute asthma needs a certain amount of sedation, although not enough to depress the respiratory center. Morphine cannot be used. My favorites are, first, chloral hydrate either orally or rectally (the capsule can be punctured with a pin for rectal use), and second, paraldehyde orally or rectally. Rectal administration of paraldehyde does not 
keep the odor from the room, since this drug is excreted through the respiratory tract. Equal parts of ether and olive oil rectally can be used to put the patient to sleep. As Dr. Sheldon mentioned a minute ago, when the patient is receiving an anesthetic, one usually does not have to worry about asthma. Large doses of barbiturates must be used with caution. Whereas morphine is contraindicated, Demerol is worth consideration in selected cases, e.g., when the patient cannot tolerate other sedatives, or if they have been ineffective.

Moderator Sheldon: Dr. Rose, will you make a brief statement with regard to gamma globulin and antibiotic and chemotherapeutic agents.

DR. Rose: The effect of gamma globulin in increasing the resistance of allergic patients is not established. The rationale does not seem to be clear. The antibodies involved in the production of the cases of true extrinsic asthma are found in the gamma globulin fraction. Gamma globulin is innocuous, if one wants to give it. It is not a sensitizing agent, as it is native to human beings.

The subject of antibiotics and chemotherapeutic agents is a big one. Briefly, there are 3: the sufonamides, bactericidal agents, and bacteriostatic agents. The best bactericidal agent is penicillin; unfortunately it may sensitize the patients, so it should not be used if one can help it. It should never be instilled into an antrum, given as a lozenge, or put on the skin. It is least innocuous when given by mouth. The usual blood levels obtained are adequate to control most infections.

Any one of the newer bacteriostatic agents can be used. Despite initial adverse reports, we still believe that chloramphenicol is one of the most effective.

Moderator Sheldon: Thank you. I should like to emphasize at this point that, when any medication is given to a patient, there is the risk of an allergic response. Dr. Sherman alluded to the subject in relation to aspirin, and Dr. Rose alluded to it in relation to penicillin. Any other medication that might be given to an elderly asthmatic patient is also capable of producing allergic shock, asthma, skin eruptions, and even death. Therefore, it is extremely important to take a careful history before any medication is given to an allergic patient (this would apply to any person). It takes only one minute to ask the patient whether or not he has had the drug you wish to prescribe; and if he has had it, whether or not any symptoms resulted from its administration. If there were symptoms, it is unwise to use that particular medication, or one from a closely related group of drugs.

I had in mind asking, unbeknown to the speakers, a number of other questions to complete the picture as we see it in asthmatic patients. However, our chairman allotted us an hour and forty-five minutes. In the time left, I should like to ask for questions from the floor, and then to summarize what we have attempted to cover.

Are there any questions from the floor?

SPEAker: I am Dr. Salteforis, a Public Health Officer in Sweden.

This is about the cause of asthma. I should like the opinion of the panelists. During the past two years, I have had several cases of bronchial asthma, and among them 2 cases of status asthmaticus immediately following shock. The 
shock was caused by a traffic accident. The patients were 2 middle-aged women, 60 or 65 years old. (From the point of view of geriatrics, I consider that middle age.) We had no past history, and nothing relevant to assist in finding the explanation.

I would be interested to know whether, in America, you think that shock could be the cause of asthma. Could a relation be found between the two clinically?

Moderator Sheldon: I believe the question is: Do the panelists believe that shock can be the trigger mechanism in inducing status asthmaticus? Dr. Cooke?

Dr. Cooke: I have never seen a case like those cited by our friend from Sweden. I suppose it is possible. We have always thought that shock might be a factor in the precipitation of an attack in those who have previously had asthmatic manifestations. That it might be the cause of a true asthmatic condition would perhaps be open to question. I would say that these persons had the dyspnea characteristic of an asthmatic attack. Whether or not it could properly be called asthma is debatable, because I did not understand the basis for the diagnosis of asthma. However, this occurrence is outside of my experience. I have never seen anything happen as promptly as that.

Moderator Sheldon: Are there any other comments from the members of the panel?

SPEAKER: Should breathing exercises or diaphragmatic belts be used?

Moderator Sheldon: A question is asked about breathing exercises and the use of emphysema belts. This has been popularized and used extensively in England. I shall attempt to answer the question from my own experience.

Theoretically, it should be very effective. From the practical standpoint, it is difficult to have a patient carry through breathing exercises effectively. Our results have been poor. For that reason, I have yet to see any measurable evidence-if one is to use pulmonary function tests as a guide-that belts and breathing exercises produce improvement in symptoms.

Some patients have said that they felt better after using a belt, or breathing exercises, but there was no change in their pulmonary function.

Another question?

SPEAKER: (A question is asked with regard to the cost of ACTH preparations.)

Moderator Sheldon: Will some member of the panel answer the question with regard to the cost of ACTH preparations.

Dr. Rose: A question arises as to the difference in cost of the various ACTH preparations. The first reason is that, if a manufacturer can get more for his product, he obviously will do so. The second reason is that, until recently, disagreement existed among manufacturers as to the unitage, which is now standard. As I understand it, all preparations should cost approximately the same.

Moderator Sheldon: In other words, you are paying on the basis of unitage whether you buy for four dollars or for eight.

Another question?

SPEAKer: (Question inaudible.)

Moderator Sheldon: Dr. Hampton, will you answer the question. 
DR. Hampton: The question concerned the speed of injection of aminophylline. It is of foremost importance to avoid a generalized vasomotor collapse. When aminophylline is given by syringe, one should administer only 1 cc. per minute until a dose of $250 \mathrm{mg}$. in $10 \mathrm{cc}$, , or $500 \mathrm{mg}$. in $20 \mathrm{cc}$., is given. An intravenous drip with glucose can be used. Slow administration is better in the long run, although less rapid in its action. The glucose may have a synergistic effect on the aminophylline.

Moderator ShELdon: I should like to take one minute to summarize. I hope that I speak for the panel. What I have heard today suggests that, in general, the management of the elderly asthmatic patient has many problems in common with the management of other allergic patients. There are certain peculiarities in these patients after the age of 40 years, in addition to sensitivity, for which there is no specific treatment. Infection plays an important role.

When an asthmatic patient undergoes a procedure that may cause shock (e.g., a surgical operation) and he has received corticosteroids or corticotropin within the preceding six months, he should again receive corticosteroids, preferably starting twenty-four hours before operation and continuing through the first few postoperative days.

In the elderly asthmatic patient, a factor not present in young persons may be added. That is the factor of retirement and of incapacity. I had expected to cover this particular phase with the panel, but can now only briefly mention the importance of having the elderly asthmatic patient occupied in some way.

Psychosomatic factors are a problem in all asthmatics, and particularly in elderly subjects.

A number of medications can be used, and used safely. The panel has attempted to discuss them in the order of their choice. 